\title{
FILTERING OF MAGNETIC RESONANCE IMAGES USING AN ADAPTIVE HYBRIDIZED FUZZY FILTER
}

\author{
R.Sindhiya Devi \\ Research Scholar, Department of Electronics and Communication Engineering, \\ Kalasalingam University, Krishnankoil, Srivilliputhur,Tamilnadu, India \\ sindhiyadevi14@gmail.com \\ Dr.B.Perumal \\ Assistant Professor, Department of Electronics and Communication Engineering, \\ Kalasalingam University, Krishnankoil, Srivilliputhur,Tamilnadu, India \\ palanimet@gmail.com \\ Dr.M.Pallikonda Rajasekaran \\ Associate Professor, Department of Electronics and Communication Engineering, \\ Kalasalingam University, Krishnankoil, Srivilliputhur,Tamilnadu, India \\ mpraja@klu.ac.in
}

\begin{abstract}
An image should be processed carefully in order to get its optimized form. The main problem during the acquisition of the images through electronic ways and means is the noise that arises. The denoising capability of the median filter is better compared to most of the available filters and hence we look into it. The elevated version of median is the adaptive median filtering which includes spatial information for the conservation of every detail and also the reduction of noise. In case of uncertainty, Fuzzy logic works well. Here, we filter the impulsive salt and pepper noise in MRI images. A median filter that uses Fuzzy logic and the adaptive median filter are compared with the modified Type 2 Fuzzy filter that is suggested for the denoising approach. Their outputs are compared to show its outperformance by using the PSNR, and Structural Similarity index parameters.
\end{abstract}

Keywords: Denoising; DBAMF; NAFSM; AHFF; Impulse noise.

\section{Introduction}

Mankind has different senses to understand about the occurrences and among them our visual perception is at its leading edge. However, we can get only a limited band of visibility of the electromagnetic (EM) spectrum. But the devices that are used for the acquisition of images could pertain to a large extent in the EM spectrum waves. This brings about the importance of such digital imaging devices that are significant to various applications in the field of digital image processing.

The quality of an image can be improved so as to get its enhanced form by means of the highly usable field called Image Processing. For the conversion of the raw images into highly applicable images, we find more number of processes. These images can be used in many digital applications of various fields. But the problem is the presence of noise in such raw images that arises during acquisition or communication. Noises are the major disturbances that completely diminish the quality of the image. These noises may be of additive type or multiplicative type in general and should be reduced in such a way to improvise the image quality.

In the medical field, the imaging technologies that are being used in common are computed tomography (CT), magnetic resonance imaging (MRI) and positron emission tomography (PET) scanning technologies. The customary noises present in these types of images include the impulse noise which is the salt and pepper noise and the randomly occurring Gaussian noise.

There exist a number of methodologies for the removal of such noises. We call them as filters as it filter out the noise. We find lots and lots of filters literally which differ in its time complexity and its procedure [Rohini et al. (2013)]. In our paper, we have obtained a goal which is to investigate different filters for its efficiency and its reliability towards the work finding and removing the impulsive salt and pepper noise present in MRI brain images. 


\section{Existing Adaptive Filters}

We have considered the filters for the noise removal of impulsive salt and pepper noise from the MRI brain images with tumor. The complete explanation and procedure for those filters are given below:

\subsection{Decision based adaptive median filter}

As we know, the median filter replaces the current pixel value with the median value the corresponding window. The adaptive median filter is somewhat vary from the conventional median filter with the fact that the size of the corresponding window considered will be increased stepwise if the given condition is not satisfied. In cases of satisfied condition, the median value is given to the current pixel as in case of normal median filter.

The impulse noise is normally random in nature that its concentration varies from pixel to pixel. In some portions, their concentration exceeds the average level of noises which means that the noise gets crowded in such portions. In some cases, it is less crowded which does not need a complex filter for the removal of noise. Others may have no such noises to remove. So, a worthy way to remove such random noises is to get the filter's size adjusted depending upon the noise concentration. Since the median filter used adjusts its size itself depending on the noise concentration, this could be called as an adaptive median filter [Suman Shrestha, (2014)]. So, many authors show their interest in finding works for the adaptation of the filter to noise. Generally, the starting window size to be considered is $3 \times 3$. This size gets increased until the condition is satisfied or till its maximum size is reached. The type of adaptive median filter that we are considering is the decision based adaptive median filter. We can understand the complete procedure of the decision based adaptive median filter from the following flowchart:

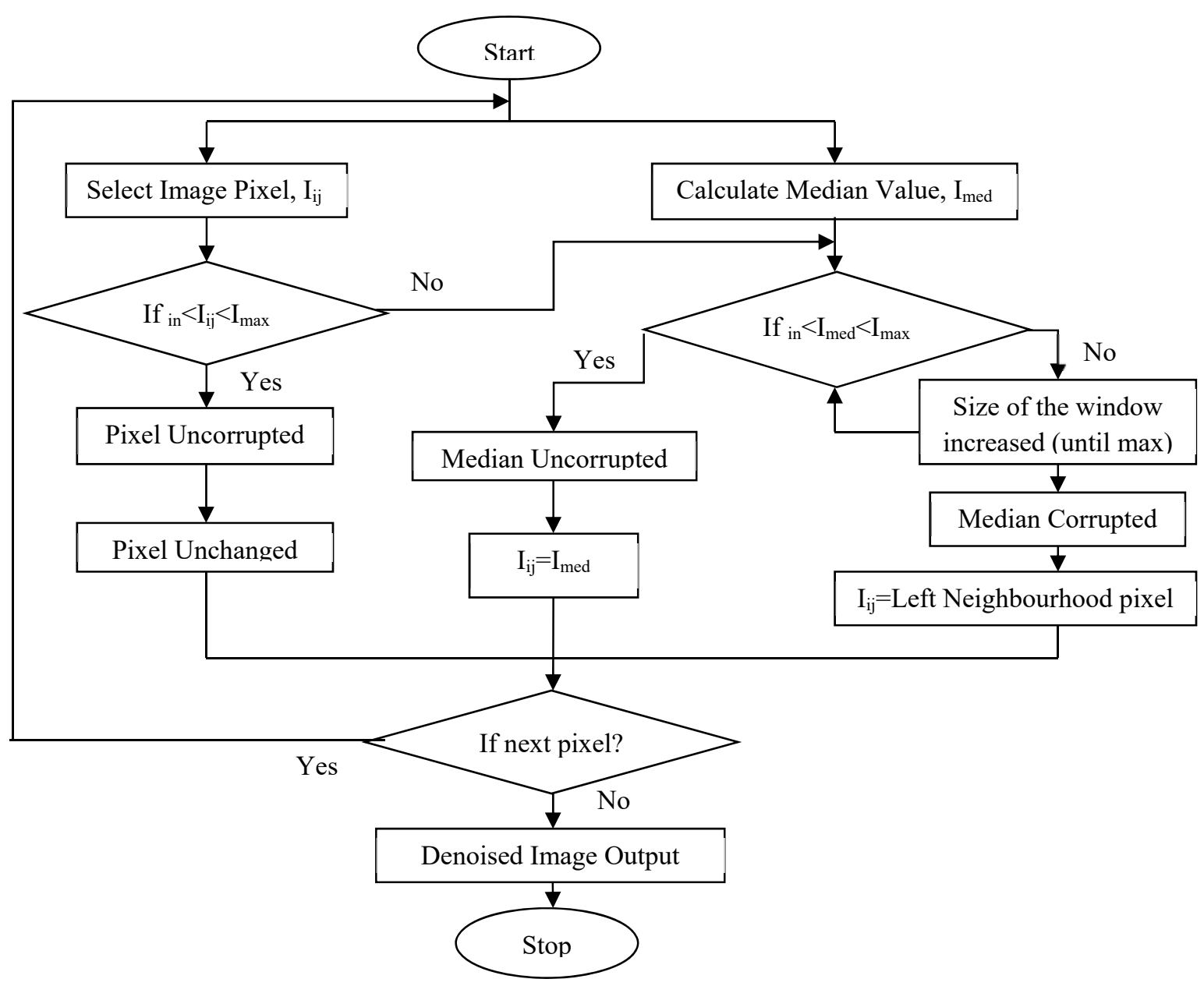

Fig. 1. Flowchart for Decision Based AMF

This type of adaptive median filter is based on the decision taken by the filter. This filter first checks the current pixel to know about its corruption status. If the pixel value is not more than the maximum value and not less than the minimum value of the corresponding window, then the current pixel is said to be uncorrupted. Otherwise, it is said to be corrupted. 
The above flowchart describes the procedure of the decision based adaptive median filter which involves three deciding factors. For a current pixel, Iij the filter first checks and decides whether the current pixel is corrupted or not. If it is found to be uncorrupted, the filter does not change the pixel value, otherwise it goes to check the median value. Now, if the median of the corresponding window of size $3 \times 3$ is checked for its corruption status. If it is uncorrupted, then the current pixel value will be replaced by the median value. If not so, the window size gets increased and if it attains the maximum value with corrupted median, then the current pixel value will be replaced by its left neighborhood that is already filtered out. This process happens until the last pixel is verified.

\subsection{Noise adaptive fuzzy switching median filter}

Noise adaptive fuzzy switching median (NAFSM) filter is an advanced form of filter combining the processes of both adaptive median filter and fuzzy switching median filter. This filter is also adaptive to the noise so that the size of the window could be increased in accordance with the corruption concentration [Arezou Banitalebi Dehkordi and Abu-Bakar, (2015)]. Also, this filter can be switched to the corrupted pixels only so that the filtering time could be reduced. In case of uncertainty of information while detecting the corrupted pixels, deciding factor lies with the fuzzy reasoning.

The NAFSM filter performs the process of finding out the corrupted pixels first before locating them. When it detects a corrupted pixel, it starts its filtering operation, otherwise no alteration will be done in that specific pixel and it jumps to the next pixel.

To find out the corrupted pixels, the NAFSM filter uses a histogram in order to find out the density of the noise. In this histogram, the filter searches for two local maximum peaks moving from both ends of the histogram. This search is sensitive to direction and it moves towards the centre of the histogram. These two local maximum peaks are useful in finding the possibility of corrupted pixels in the image. After this, it creates a mask to locate those corrupted pixels.

The next step is to filter out the noisy pixels present in the image. This is done by replacing the values of corrupted pixels. The starting window size is considered as $3 \times 3$ and the number of uncorrupted pixels is counted which is used to estimate the median. If there is not even a single uncorrupted pixel present in this window, then window size is to be increased until the condition is satisfied or to be stopped when it reaches $7 \times 7$ even if there are no uncorrupted pixels. Here it chooses the first four pixels forming the upper left diagonal for the perfect estimation of the median.

Once when the median is estimated, the local information is to be found. This could be done by calculating the maximal absolute difference of luminance which sets the corrupted pixels to the maximum of 255 and the remaining values for the uncorrupted pixels. This extraction of the local information is performed by using fuzzy reasoning. To the end, the noisy pixels are restored by a correction term with a weight provided by the fuzzy membership function.

\section{Adaptive Hybridized Fuzzy Filter}

The Adaptive Hybridized Fuzzy Filter (AHFF) is an adaptive filter with the hybrid form of Type 1 and Type 2 fuzzy filters. There are two stages in this filtering process that includes the distribution of corrupted and uncorrupted pixels using Type 2 fuzzy sets and the de-noising of the corrupted pixels employing Type 1 fuzzy sets.

\subsection{Distribution of pixels}

The distribution of pixels as corrupted and uncorrupted consists of the following three steps:

- Step 1: To estimate the set of neighbourhood pixels.

The set of neighbourhood pixels of a currently selected pixel, pij in an image having half of its window size as $\mathrm{H}$ can be computed by using the formula,

$$
B_{i j}^{H}=\left\{p_{i+a, j+b} \forall a, b \in[-H, H]\right\}
$$

where its window size is $(2 \mathrm{H}+1) \times(2 \mathrm{H}+1)$

- Step 2: To estimate the set of neighbourhood pixels.

The set of neighbourhood pixels of a currently selected pixel, $p_{i j}$ in an image having half of its window size as $\mathrm{H}$ can be computed by using the formula,

where,

$$
\dot{M}_{i j}^{(H)}=\left\{\left(p_{i j}, \mu_{M_{i j}^{H}}\right), \mu_{M_{i j}^{H}}\left(p_{i j}, \mu_{M_{i j}^{H}}\right) \forall p_{i j} \in U\right\}
$$

$$
0<\mu_{M_{i j}^{(H)}}, \mu_{\dot{\mathrm{M}}_{i j}^{(H)}}\left(p_{i j}, \mu_{M_{i j}^{(H)}}\right) \leq 1
$$


The value of mean is then calculated by using the Eq. (3),

$$
\vartheta_{i j}^{(H, k)}=m_{k}\left(B_{i j}^{H}\right), k=1,2, \ldots, x
$$

where, $\mathrm{mk}$ - mean of $\mathrm{k}$ middle

The value of the variance can be distinct or same. Hence we can use Eq. (4) for distinct variance and Eq. (5) for the same variance

$$
\begin{aligned}
& \sigma_{i j}^{(H)}=m_{k}\left(\Omega_{i j}^{H}\right) \\
& \quad \sigma_{i j}^{(H)}=m_{k}\left(\Omega_{i j}^{H}\right), k=1,2, \ldots, x
\end{aligned}
$$

Here, the parameter $\Omega \mathrm{ij}$ can be estimated using the following equation:

$$
\Omega_{i j}^{(H)}=\left\{\left|b_{n}-\vartheta_{a v g}\right| \forall b_{n} \in B_{i j}^{H}\right\}
$$

where,

$$
\vartheta_{\text {avg }}=\frac{1}{x} \sum_{1}^{x} \vartheta_{i j}^{(H, x)}
$$

Finally, the membership matrix is obtained as follows:

with its size $\varkappa \times \mathrm{n}$.

$$
\Delta_{i j}=\left[\begin{array}{cccc}
\mu_{M_{i j}^{(H, 1)}}\left(b_{1}\right) & \mu_{M_{i j}^{(H, 1)}}\left(b_{2}\right) & & \mu_{M_{i j}^{(H, 1)}}\left(b_{n}\right) \\
\mu_{M_{i j}^{(H, 2)}\left(b_{1}\right)} & \mu_{M_{i j}^{(H, 2)}}\left(b_{2}\right) & \cdots & \mu_{M_{i j}^{(H, 2)}}\left(b_{n}\right) \\
\vdots & \ddots & \vdots \\
\mu_{M_{i j}^{(H, x)}}\left(b_{1}\right) & \mu_{M_{i j}^{(H, x)}}\left(b_{2}\right) & \cdots & \mu_{M_{i j}^{(H, x)}}\left(b_{n}\right)
\end{array}\right]
$$

- $\quad$ Step 3: To classify the pixels as uncorrupted and noisy.

The estimation of the threshold is necessary in order to classify and distribute the pixels as corrupted and uncorrupted pixels. This estimation can be done by using the Eq. (9).

$$
T h=\wedge\left(\mathrm{v}\left(\Delta_{i j}\right)\right)
$$

where, $\wedge$ - min operator (T-norm)

$$
\checkmark \text { - max operator (S-norm) }
$$

We should find the membership value for each and every pixel to classify them. This can be done by using Eq. (10).

$$
\mu_{M_{i j}^{(H)}}=\frac{\sum_{k=1}^{x} \Delta_{i j}}{x} \forall i=1,2, \ldots, n(10)
$$

Now, the membership values of every pixel are compared with the threshold value. If membership value of a specific pixel is larger when comparing to the threshold, then it is said to be an uncorrupted pixel. If not so, it is classified under corrupted pixel and is sent for de-noising.

\subsection{Removal of Noise}

The corrupted pixels that are obtained from the previous stage are brought here for the removal of noise. In doing so, the major importance lies with the weights of the uncorrupted pixels given by Type 1 fuzzy set. We consider the uncorrupted pixel set as the current fuzzy set where we map each and every element to [0, 1]. The noise removed pixels can be obtained by using the equation (11) given below:

$$
\begin{aligned}
& p_{\text {denoised }}=\frac{\sum \forall f_{1} \in F^{w_{i} f_{i}}}{W} \\
& W=\sum_{i=1}^{\delta} w_{i}
\end{aligned}
$$

where, wi - weight of the noise free pixel in the place ' $i$ '

$\delta$ - Noise free pixels' population

$\mathrm{W}$ - Terminal figure for normalization

This procedure stops at once, when the value of $\zeta$ goes beneath $0.05 \%$. It is nothing but the noise free pixels' representation in percentage. It is obtained by using the following equation:

$$
\zeta=\frac{n p^{n e x t}-n p^{p r e v}}{A \times B}
$$

Here, $n p^{\text {next }}$ and $n p^{\text {prev }}$ are the corrupted pixels of successive and previous iterations. AxB denotes the size of the window. 


\section{Results and Discussion}

We have accomplished the above three specified algorithms in four brain tumor MRI images of size 512 x 512 . Those input brain tumor MRI images are presented in the figure 2.
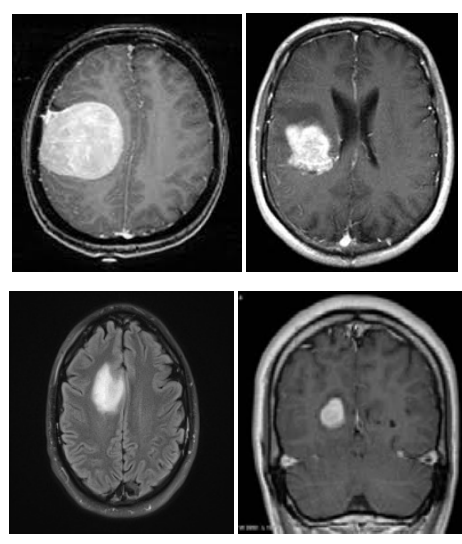

Fig. 2. Input Images

We have inserted some impulsive salt and pepper noises to the input images thereby investigating their efficiencies. We have considered the percentage of noise density randomly within $0 \%$ and $50 \%$ as we know that the medical images will have only less density of noises. The filtered outputs of the inputs along with the four given input images at $3 \%, 6 \%, 12 \%, 24 \%, 33 \%$ and $47 \%$ impulsive salt and pepper noise are showcased in figure 3 , figure 4 , figure 5 and figure 6 respectively.

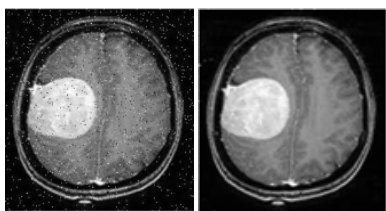

(a)

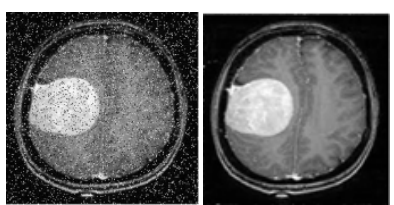

(c)

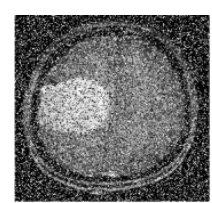

(e)

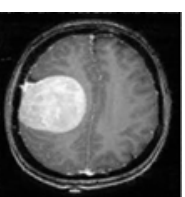

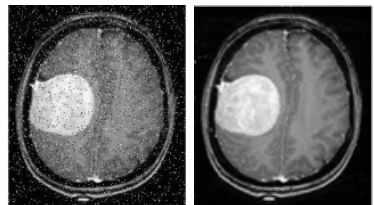

(b)

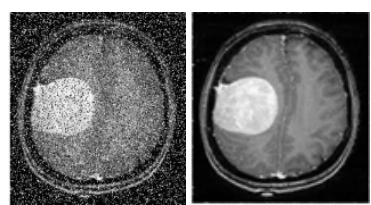

(d)

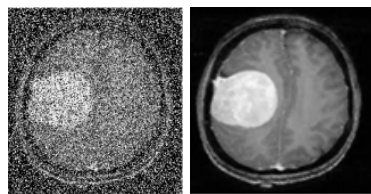

(f)

Fig. 3. Image 1: (a) at 3\% noise, (b) at $6 \%$ noise, (c) at $12 \%$ noise, (d) at $24 \%$ noise, (e) at $33 \%$ noise, (f) at $47 \%$ noise with their filtered outputs. 


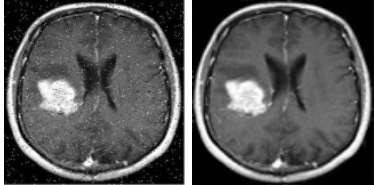

(a)

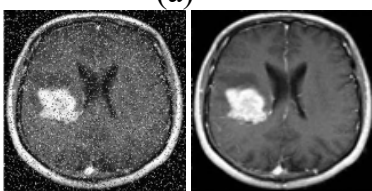

(c)

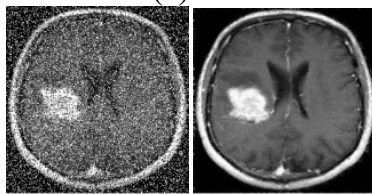

(e)

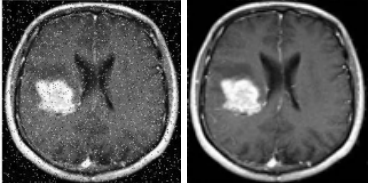

(b)

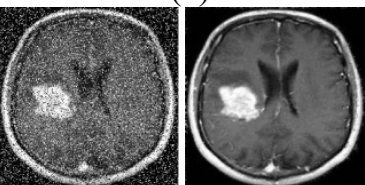

(d)

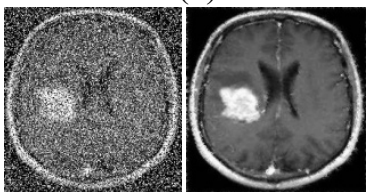

(f)

Fig. 4. Image 2: (a) at 3\% noise, (b) at $6 \%$ noise, (c) at $12 \%$ noise, (d) at $24 \%$ noise, (e) at $33 \%$ noise, (f) at $47 \%$ noise with their filtered outputs.
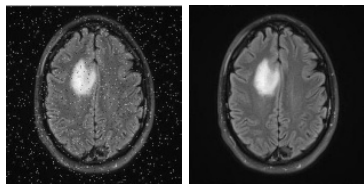

(a)

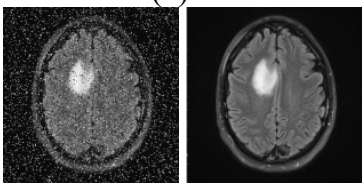

(c)

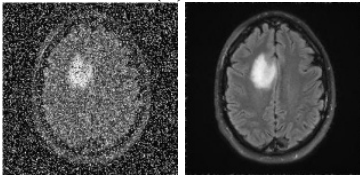

(e)

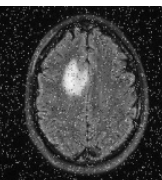

(b)

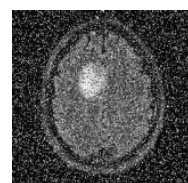

(d)

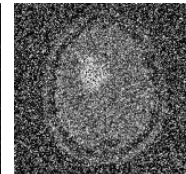

(f)
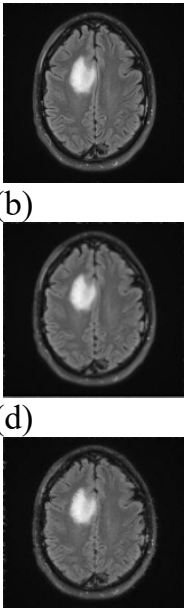

Fig. 5. Image 3: (a) at 3\% noise, (b) at $6 \%$ noise, (c) at $12 \%$ noise, (d) at $24 \%$ noise, (e) at $33 \%$ noise, (f) at $47 \%$ noise with their filtered outputs.

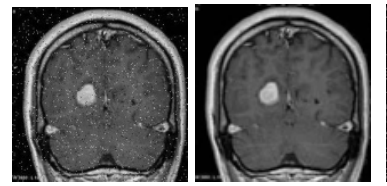

(a)

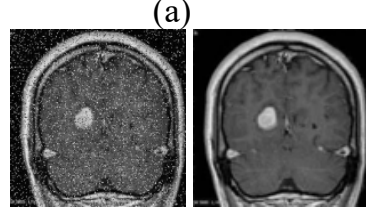

(c)

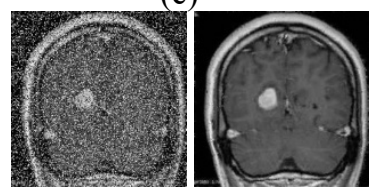

(e)

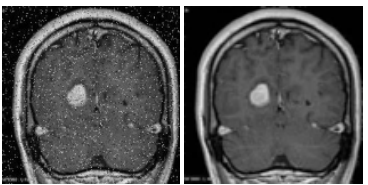

(b)

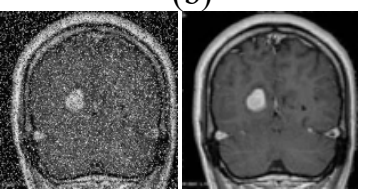

(d)

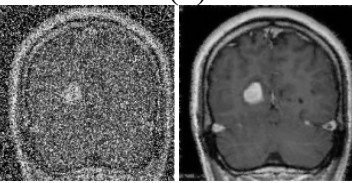

(f)

Fig. 6. Image 4: (a) at 3\% noise, (b) at $6 \%$ noise, (c) at $12 \%$ noise, (d) at $24 \%$ noise, (e) at $33 \%$ noise, (f) at $47 \%$ noise with their filtered outputs. 
In our paper, the results of these filters have been investigated with Peak Signal to Noise Ratio (PSNR) and Structural Similarity Index (SSIM). The larger the values of PSNR and SSI, the better will be their performances. The PSNR specifies the ratio of maximal possibility value of an image to the value of the corrupted pixels which affects the efficiency. The outcomes of the PSNR values of Adaptive Median Filter based on Decision, Noise Adaptive Fuzzy Switching Median Filter and the Adaptive Hybridized Fuzzy Filter are exhibited in the Table I given below:

Table 1. PSNR Comparison

\begin{tabular}{|c|c|c|c|c|}
\hline Images & Noise (\%) & DBAMF & NAFSM & AHFF \\
\hline \multirow{6}{*}{ I } & 3 & 32.8182 & 45.7602 & 47.1681 \\
\hline & 6 & 32.8156 & 43.2395 & 45.4415 \\
\hline & 12 & 32.8284 & 42.5008 & 43.3567 \\
\hline & 24 & 32.8194 & 39.5653 & 40.3753 \\
\hline & 33 & 32.8099 & 36.2997 & 38.5288 \\
\hline & 47 & 32.7583 & 34.0997 & 36.6353 \\
\hline \multirow{6}{*}{ II } & 3 & 32.2703 & 45.2408 & 46.6751 \\
\hline & 6 & 32.2789 & 44.2846 & 44.9397 \\
\hline & 12 & 32.2845 & 41.5194 & 42.6695 \\
\hline & 24 & 32.2983 & 38.9628 & 39.9742 \\
\hline & 33 & 32.2410 & 37.5883 & 38.2143 \\
\hline & 47 & 32.2918 & 35.9359 & 36.0521 \\
\hline \multirow{6}{*}{ III } & 3 & 33.5324 & 45.9326 & 50.8169 \\
\hline & 6 & 33.4992 & 44.7644 & 47.8721 \\
\hline & 12 & 33.4926 & 42.8165 & 44.4443 \\
\hline & 24 & 33.437 & 38.8355 & 41.3941 \\
\hline & 33 & 33.4228 & 37.3404 & 39.4992 \\
\hline & 47 & 33.3436 & 33.2537 & 37.7556 \\
\hline \multirow{6}{*}{ IV } & 3 & 32.5362 & 45.1281 & 46.1308 \\
\hline & 6 & 32.5331 & 43.4124 & 44.8399 \\
\hline & 12 & 32.5402 & 40.8989 & 42.4258 \\
\hline & 24 & 32.5905 & 38.3896 & 39.8075 \\
\hline & 33 & 32.5949 & 36.9921 & 38.1633 \\
\hline & 47 & 32.4697 & 32.4681 & 36.1062 \\
\hline
\end{tabular}

Similarly, the Structural Similarity Index (SSIM) is a measure for investigating the quality of the image by comparing it with the reference or the original image. It shows the similarity between images based on perception depending on the luminance, contrast and the structure. The outcomes of the SSIM values of Adaptive Median Filter based on Decision, Noise Adaptive Fuzzy Switching Median Filter and the Adaptive Hybridized Fuzzy Filter are exhibited in the Table II given below: 
Table 2. SSIM Comparison

\begin{tabular}{|c|c|c|c|c|}
\hline Images & Noise (\%) & DBAMF & NAFSM & AHFF \\
\hline \multirow{6}{*}{ I } & 3 & 0.7608 & 0.9814 & 0.9947 \\
\hline & 6 & 0.7604 & 0.9751 & 0.9920 \\
\hline & 12 & 0.7584 & 0.9513 & 0.9870 \\
\hline & 24 & 0.7509 & 0.9325 & 0.9696 \\
\hline & 33 & 0.7453 & 0.9121 & 0.9497 \\
\hline & 47 & 0.7296 & 0.8543 & 0.8924 \\
\hline \multirow{6}{*}{ II } & 3 & 0.7804 & 0.9825 & 0.9929 \\
\hline & 6 & 0.7797 & 0.9656 & 0.9904 \\
\hline & 12 & 0.7783 & 0.9641 & 0.9848 \\
\hline & 24 & 0.7693 & 0.9405 & 0.9679 \\
\hline & 33 & 0.7635 & 0.9245 & 0.9476 \\
\hline & 47 & 0.7497 & 0.8639 & 0.8968 \\
\hline \multirow{6}{*}{ III } & 3 & 0.7333 & 0.9771 & 0.9962 \\
\hline & 6 & 0.7318 & 0.9674 & 0.9928 \\
\hline & 12 & 0.7281 & 0.9592 & 0.9848 \\
\hline & 24 & 0.7220 & 0.9304 & 0.9651 \\
\hline & 33 & 0.7180 & 0.9148 & 0.9446 \\
\hline & 47 & 0.7087 & 0.8548 & 0.8945 \\
\hline \multirow{6}{*}{ IV } & 3 & 0.8015 & 0.9716 & 0.9959 \\
\hline & 6 & 0.8006 & 0.9863 & 0.9939 \\
\hline & 12 & 0.7994 & 0.9745 & 0.9888 \\
\hline & 24 & 0.7930 & 0.9508 & 0.9736 \\
\hline & 33 & 0.7873 & 0.9322 & 0.9562 \\
\hline & 47 & 0.7724 & 0.8491 & 0.9058 \\
\hline
\end{tabular}

The comparison of the above specified three adaptive algorithms in terms of PSNR and SSIM are plotted in figure 7 and figure 8 respectively.

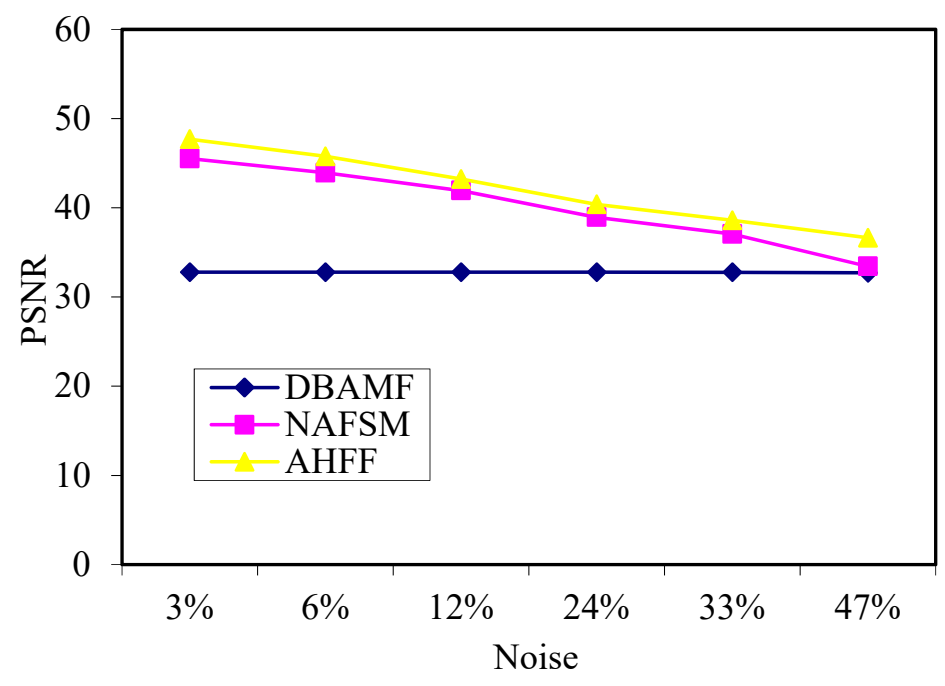

Fig. 7. Comparison of Peak Signal to Noise Ratio 


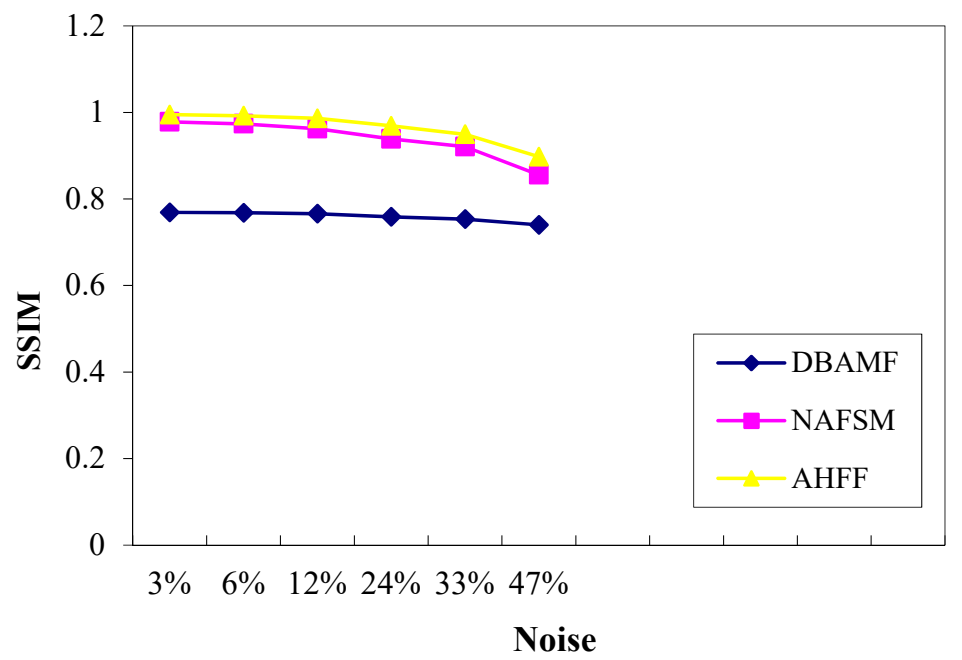

Fig. 8. Comparison of Structural Similarity Index

From the exploration, we found that the filter with fuzzy logic is better when comes to uncertainty cases. The Adaptive Hybridized Fuzzy Filter that uses Type 2 and Type 1 fuzzy filters outstand the performance of NAFSM. It shows about $96.5 \%$ structural similarity to the original image. Similarly, the similarity of NAFSM filter is $93.8 \%$ and the decision based Adaptive Median filter is $75.9 \%$ similar to the original image.

\section{Conclusion}

Adapting to the nature of the composition of noises is very important during the process of filtering. We have investigated two adaptive methods of filtering methodologies and suggested a hybrid algorithm that is also adaptive in nature with fuzzy logic sets. This hybrid algorithm gives better results when compared to the other two methods. The outcomes of the three adaptive techniques are measured with the terminologies of PSNR and SSIM. The average PSNR obtained for the suggested AHFF algorithm is 42.0536 and its structural similarity to the original images is $96.5 \%$ which is comparatively greater than the other two filters.

\section{References}

[1] Mansi Pathak.; Sinha, G. R. (2014): A Survey of Fuzzy Based Image Denoising Techniques. IOSR Journal of Electronics and Communication Engineering, 9(4), pp. 27-36.

[2] Suman Shrestha. (2014): Image Denoising using New Adaptive Based Median Filter. Signal \& Image Processing: An International Journal, 5(4), pp. $1-13$.

[3] Arezou Banitalebi Dehkordi.; Abu-Bakar, A. R. (2015): Adaptive Fuzzy Switching Noise Reduction Filter for Iris Pattern Recognition. Jurnal Teknologi, 73(1), pp. $27-33$.

[4] Mojtaba Ahmadieh Khanesar.; Mohammad Teshnehlab.; Erdal Kayacan.; Okyay Kaynak. (2010): A Novel Type-2 Fuzzy Membership Function: Application to the Prediction of Noisy Data. IEEE International Conference on Computational Intelligence for Measurement Systems and Applications.

[5] Rohini, R.; Varade, M. R.; Archana, B. (2013): A Survey on Various Median Filtering Techniques for Removal of Impulse Noise from Digital Images. International Journal of Advanced Research in Computer Engineering \& Technology, 2(2).

[6] Youlian Zhu.; Cheng Huang. (2012): An Improved Median Filtering Algorithm for Image Noise Reduction. Physics Procedia, Elsevier, 25 , pp. $609-616$.

[7] Murugeswari, P.; Manimegalai, D. (2011): Noise Reduction in Color image using Interval Type-2 Fuzzy Filter (IT2FF). International Journal of Engineering Science and Technology, 3(2), pp. $1334-1338$.

[8] Oscar Castillo.; Mauricio A. Sanchez.; Claudia I. Gonzalez.; Gabriela E. Martinez. (2017): Review of Recent Type-2 Fuzzy Image Processing Applications. Information, 8(97), pp. $1-18$.

[9] Sin Hoong Teoh.; Haidi Ibrahim. (2012): Median Filtering Frameworks for Reducing Impulse Noise from Grayscale Digital Images: A Literature Survey. International Journal of Future Computer and Communication, 1(4), pp. $323-326$.

[10] Jerry M. Mendel.; Robert I. Bob John. (2002): Type-2 Fuzzy Sets Made Simple. IEEE Transactions on Fuzzy Systems, 10(2), pp. 117 127.

[11] Zhou, Z. (2012): Cognition and removal of impulse noise with uncertainty. IEEE Transactions on Image Processing, 21(7), pp. 31573167.

[12] Rojas, R.; Rodrguez, P. (2011): Spatially adaptive total variation image de-noising under salt and pepper noise. Proceedings of European Signal Processing Conference, Barcelona, Spain, 19, pp. 278-282.

[13] Lee, C. S.; Kuo, Y. H. (2000): Adaptive fuzzy filter and its application to image enhancement. Fuzzy Techniques in Image Processing, Studies in Fuzziness and Soft Computing, 52, pp. 172-193.

[14] Peixuan Zhang.; Fang Li. (2014): A new adaptive weighted mean filter for removing salt and pepper noise. IEEE Signal Processing Letters, 21(10), pp. 1280-1283.

[15] Vikas Singh.; Raghav Dev.; Narendra K. Dhar.; Pooja Agrawal.; Nishchal K. Verma. (2018): Adaptive Type-2 Fuzzy Approach for Filtering Salt and Pepper Noise in Grayscale Images. IEEE Transactions on Fuzzy Systems, 26(5), pp. 3170 - 3176.

[16] Amarjit Roy.; Lalit Manam.; Rabul Hussain Laskar. (2018): Region Adaptive Fuzzy Filter: An Approach for Removal of Random Valued Impulse Noise. IEEE Transactions on Industrial Electronics, 65(9), pp. $7268-7278$. 
[17] Weiping Zhang.; Mohit Kumar.; Jingzhi Yang.; Yunfeng Zhou.; Yihua Mao. (2019). An Adaptive Fuzzy Filter for Image Denoising. Cluster Computing, 22, pp. 14107-14124.

[18] Irpan Adiputra Pardosi.; Ali Akbar Lubis. (2019): Grayscale Image Quality Analysis Result of Noises Reduction using Adaptive Fuzzy Filter (AFF) and Spatial Median Filter (SMF) Against Image Depth Variations. Journal of Physics: Conference Series, 1361.

[19] Ghouse Mohamed.; Siddappa.; Inamurrahman Mohammed. (2017): Robust Adaptive Fuzzy Filter for Impulse Noise in Grainy Images. International Journal of Engineering and Management Research, 7(1), pp. $151-156$.

[20] Gülcan Yildiz.; Doğan Yildiz. (2018): A New Approach to Reduce Salt - Pepper Noise using a Fuzzy Set for Gray Level Images. Electric Electronics, Computer Science, Biomedical Engineerings' Meeting (EBBT), Istanbul, Turkey. 